\title{
Independent contexts for category ratings: A range-frequency analysis
}

\author{
ALLEN PARDUCCI, SUSAN KNOBEL, and CHRISTOPHER THOMAS \\ University of California, Los Angeles, California 90024
}

\begin{abstract}
Subjects were instructed to rate with a single set of categories an intermingled series of smaller squares and larger circles, establishing a separate scale of size for each domain. The major finding was that the scale established for one domain was independent of the skewing of sizes in the other domain. However, subjects were also able to combine the two domains into a single context when instructed to do so, particularly when the smallest circle was only slightly larger than the largest square. A range-frequency analysis of the rating suggests that under certain conditions the endpoints defining the subjective range depend upon the stimulus frequencies: the subjective endpoint is more extreme when the closest stimuli are presented with greater relative frequency.
\end{abstract}

A special attraction of using psychophysical stimuli in studies of category ratings is that the particular stimulus values selected for presentation constitute the primary context for judgment. For example, the same physical size is rated "small" when presented in a series of larger sizes, "large" when the other sizes are smaller. This suggests that the experimental context is isolated from preexperimental experiences with smaller or larger sizes.

Although the range-frequency theory of judgment (Parducci, 1965, 1974) was developed to explain judgments in these isolated contexts, the theory should also be useful for analyzing situations in which subjects must deal with more than one context at a time. To explore the problem of multiple contexts, the present research encourages subjects to establish separate scales for different sets of stimuli presented in the same experimental series. A pilot study used two different sets of squares, each covering the same range of sizes but with the smaller sizes presented more often in one set and the larger sizes more often in the other. The sets also differed in color and in whether they were presented on even or odd trials. Although both sets had to be rated using the same set of response categories, subjects were able to establish two different scales. However, the difference between the scales was only about half as great as when each set was presented separately. It was unclear whether this smaller difference simply reflected the difficulty of isolating the two sets of stimuli or whether confusion between the responses was also a contributing factor.

The present experiment was designed to facilitate isolation of the two sets of stimuli. Circles were sub-

Inquiries concerning this article should be addressed to Allen Parducci, Department of Psychology, University of California, Los Angeles, California 90024 . The authors wish to express their appreciation to Norman $\mathbf{H}$. Anderson for his helpful suggestions. stituted for squares on half the trials, with the smallest circle larger than the largest square. The task was to establish separate scales for circles and squares. Insofar as the same response categories would be used for both sets, some confusion of contexts would still be expected. Experimental conditions were selected to permit inference of the multiple contexts from the scales of judgment.

\section{METHOD}

\section{Instructions}

The task was to rate the size of each of a sequence of figures, squares and circles, comparing each square only with the other squares, each circle only with the other circles. Ratings were made by inserting a plug in a response box with nine holes labeled "Very Very Small," "Very Small," "Small," "Slightly Smaller than Average," "Average," "Slightly Larger than Average," "Large," "Very Large," and "Very Very Large." The instructions included the statement: "Thus, a square might be judged 'Very Large' and a circle 'Very Small,' even though the circles are all larger than the squares." For control conditions rating a single domain, squares or circles, the instructions of course made no reference to a second domain. For other control conditions in which both squares and circles were presented, subjects were instructed to "ignore the differences in shape."

\section{Stimuli}

The projected widths of the squares varied from 1.3 to $5.6 \mathrm{~cm}$ in a geometric series, with each step increasing by a factor of 1.16; these were the 12 smallest from the set of 24 squares used in a previous experiment (Parducci \& Perrett, 1971). The circles extended the series up for 12 additional steps, corresponding to the 12 largest squares of the previous experiment. Thus, the smallest circle was slightly larger than the largest square. The squares were solid black on a $75 \times 100 \mathrm{~cm}$ white background, the circles red rings on a yellow background of the same size. Both the radii and thickness of the rings increased by the same factor of 1.16, the outside diameters varying from 8 to $39 \mathrm{~cm}$, the inside diameters from 6.5 to $32 \mathrm{~cm}$. As compared with stimulus sets used in the earlier research, the largest of the present squares was much smaller relative to the lighted background area, and the smallest circle was much larger. 
Table 1

Stimulus Sets

Ranks

Domain

Squares

Circles

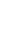

1

13

2
14

14
3
15
4
16 $\begin{array}{rr}5 & 6 \\ 17 & 18\end{array}$

8

19

8
20

9
21

10

22

11

23

12

Frequencies

\section{Distribution}

Negative

Rectangular

Positive

\begin{tabular}{|c|c|c|c|}
\hline 6 & & & 6 \\
\hline 10 & 10 & & 10 \\
\hline 16 & 14 & 14 & 6 \\
\hline
\end{tabular}

$6 \quad 10$

6
10
6

6
10
6

6

6
10
6

14

$\begin{array}{rr}14 & 16 \\ 10 & 10 \\ & 6\end{array}$

\section{Experimental Design and Procedure}

The basic experimental conditions formed a 3 by 3 factorial design, with the stimuli for each condition consisting of one of three different sets of squares and one of three different sets of circles. Table 1 gives the sizes and frequencies of the stimuli in the respective sets: these form a negatively skewed, a rectangular, and a positively skewed distribution for each of the two shapes. Four sequences of presentation were used for each of the nine conditions, randomized within the following constraints: (1) squares and circles appeared on alternate trials, (2) squares came on odd-numbered trials for half the sequences, (3) both first-order and second-order sequential dependencies among the stimuli were balanced insofar as possible, and (4) each quarter of the series was as representative as possible of the entire series. Six control conditions consisted either of one of the three sets of squares or of one of the three sets of circles. A subsidiary experiment replicated the three control conditions for squares, except that the range extended from 6 to 17 (i.e., adding 5 to each of the stimulus values for squares listed in Table 1). The two rectangular sets were presented together as an additional control, with instructions to ignore the differences in shape; a final control condition consisted of two extreme rectangular sets, the eight smallest squares and eight largest circles, each presented 10 times, again with instructions to ignore shape.

After the printed instructions were read by the subjects (along with the experimenter who read them aloud), the first $\mathbf{4 0}$ stimuli of the regular series were presented as a preview of the stimulus sets and also for practice in using the automatic recording apparatus. The entire session consisted of 200 presentations

(120 for controls presented just a single domain); the projector switched slides every $6 \mathrm{sec}$, with a switching interval of about $.5 \mathrm{sec}$. Ratings of the preview were not recorded. An experimental session lasted for about $30 \mathrm{~min}$, including instructions.

\section{Subjects}

Some 800 undergraduates participated in the experiment as part of the regular requirement for introductory psychology at UCLA. Subjects were run in groups of 10; the four groups in the same experimental condition received different orders of presentation so that a total of $\mathbf{4 0}$ subjects rated each of the nine combinations of squares and circles. Each control condition also used 40 subjects and four different orders.

\section{RESULTS}

Figure 1 shows the mean rating for each stimulus in each of the experimental sets. The numerical values were obtained by transforming the verbal categories to ranks, 1 through 9 . Since the ratings of each square were averaged across the three sets of accompanying circles, i.e., across the skewings of the irrelevant domain, the plotted differences between ratings of the same square in different sets reflect differences in skewing of the squares, the relevant domain. Similarly, the ratings of the circles reflect

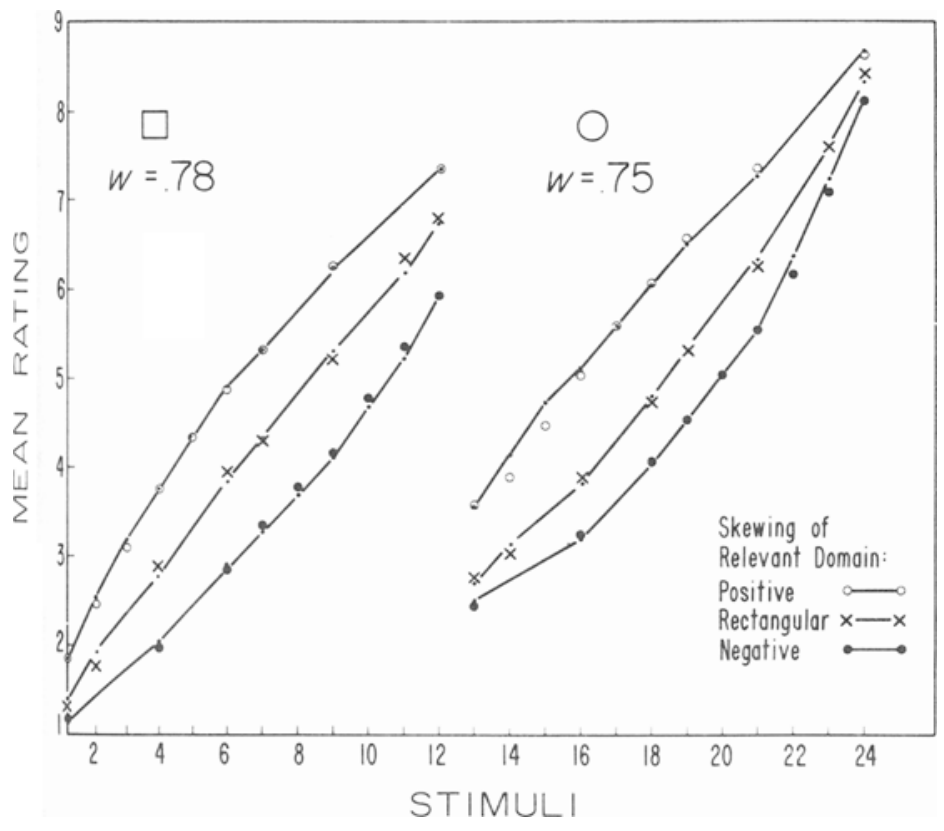

Figure 1. Contextual effects within paired domains: squares (left) and circles (right). Empirical ratings (larger symbols) for the three experimental sets averaged across the three sets of the irrelevant domain. Fitted functions (lines connecting points) derived from modified range-frequency model (Equation 2) which yielded the values of w shown. 
Figure 2. Absence of contextual effects between donains. Empirical ratings averaged across sets for the relevant domain and plotted separately for each set in the irrelevant domain.

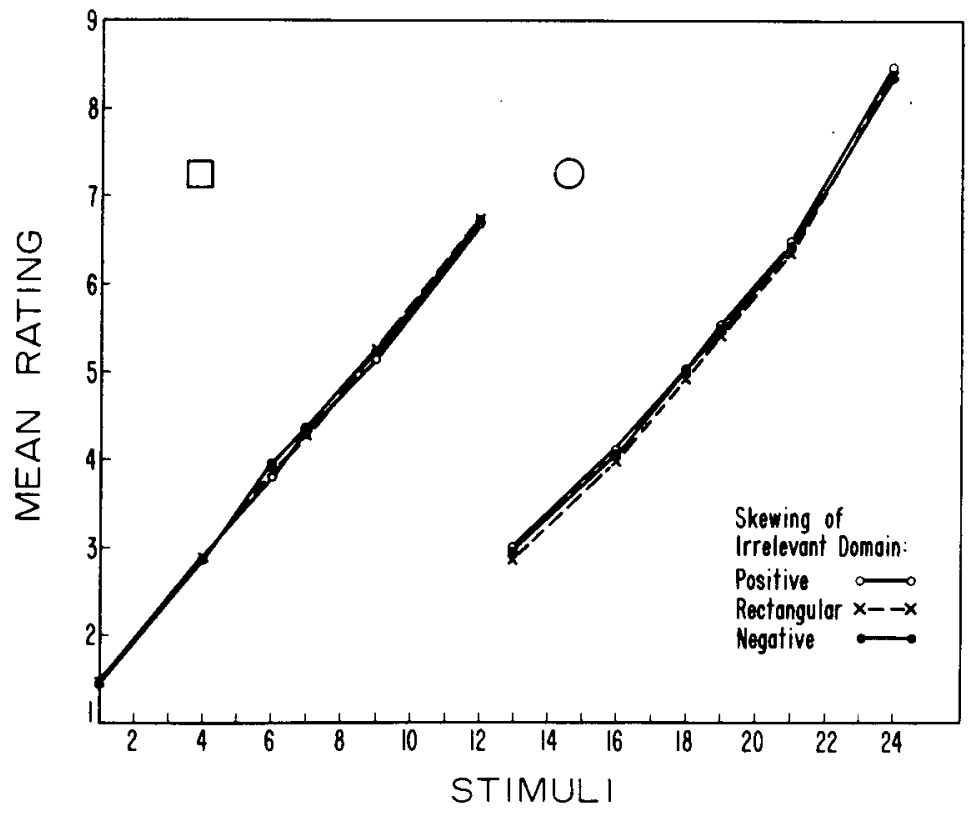

the immediate stimulus context of circles, their relevant domain. The fitted functions show what the ratings would have been if subjects had tended to equalize the frequencies within each domain in accordance with a range-frequency model. The procedure for deriving these fits is described below. The important point here is that subjects do tend to equalize frequencies within each separate domain. Figure 1 also shows that subjects can isolate the two domains to some extent: although the smallest circle is physically larger than the largest square, it is rated more than three category steps lower.

Figure 2 shows that the ratings of stimuli in one domain are not affected by the skewing of the frequency distribution of sizes in the other domain. Each point in Figure 2 represents the rating of a particular physical size averaged across the three sets in which it was presented but plotted separately for each of the three sets from the other domain. For example, the mean rating of 3.95 for Square 6 when presented with the negatively skewed set of circles was calculated by averaging the rating of Square 6 across three experimental conditions: Negative Squares with Negative Circles, Rectangular Squares with Negative Circles, and Positive Squares with Negative Circles. Thus, the closeness of the three points for each stimulus is testimony to the independence of the ratings from the skewing of the accompanying domain. Insofar as response frequencies were equalized across both domains, the surplus of "larger" categories in a negatively skewed set would have reduced the use of "larger" categories in whatever set accompanied it.

Separate analyses of variance were performed for ratings of squares and circles, in each case restricting the analysis to the six stimuli common to all three sets (because of missing subjects, all groups were reduced to $\mathrm{n}=35$ by random selection). The main effect of the skewing of the irrelevant domain was of course insignificant, $F(2,306)=.23$ and 1.24 in the respective analyses. Each analysis also provided three tests for interaction with the irrelevant domain, viz, its interaction with the relevant domain, with stimulus size, and the three-way interaction. Of these tests, only the three-way interaction for ratings of squares, Circle Set by Square Set by Square Size, was statistically significant, $F(20,1530)=2.57$, $\mathrm{p}<.01$. In this exception, ratings of the larger squares in the positive set were higher when presented with the negative set of circles, but the largest square in the negative set was rated higher when presented with the positive set of circles. This puzzling interaction did not appear in the ratings of circles, $p>.05$, and the smaller circles in the negative set were actually rated slightly higher when presented with the negative squares.

Figure 1 shows that most of the effects of the relevant context are upon the linear component of the different rating scales. However, the differences in curvature are also statistically significant. Analyses of variance performed on the quadratic component yielded values of $F(2,306)=9.45$ and 141.28 , $p<.001$, for the effects of the relevant context upon ratings of squares and circles, respectively. This result is of particular interest as a test of the modified range-frequency model to be introduced below (Equation 2). In both analyses, the effects of the irrelevant context were again insignificant, $\mathrm{F}(2,306)=.74$ and .17 .

\section{Controls}

Figure 3 shows the ratings by the six control 


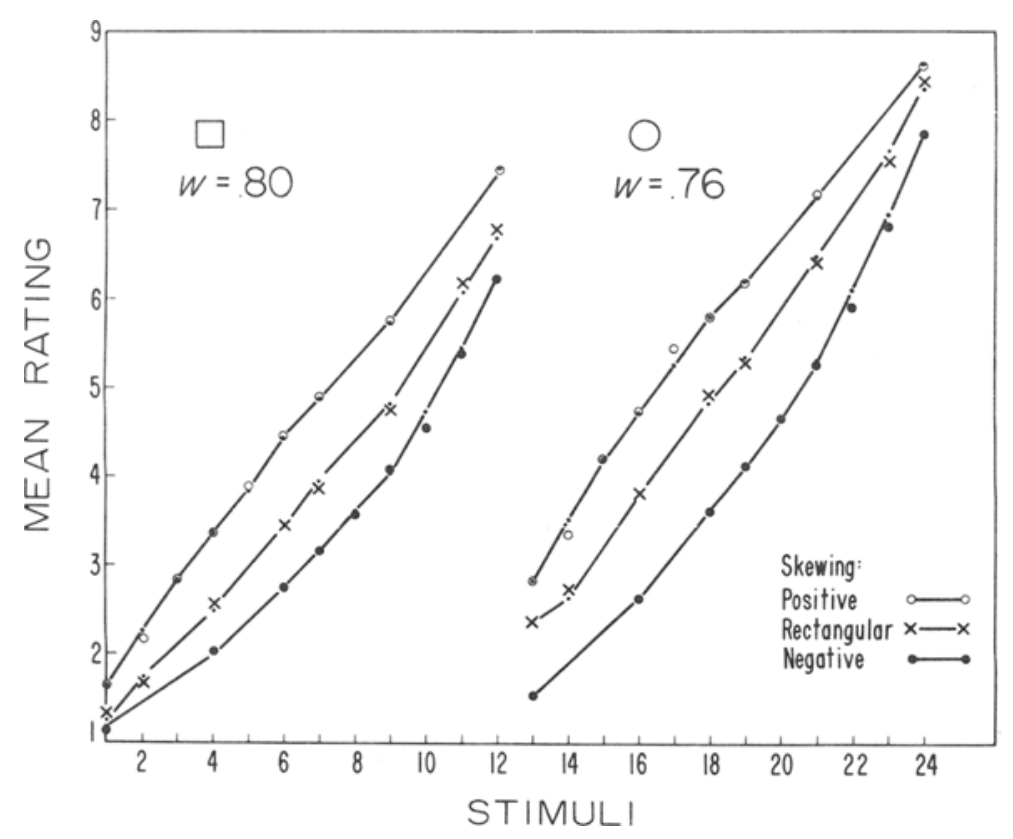

Figure 3. Contextual effects within single domains. Empirical ratings (larger symbols) for control conditions. Fitted functions (lines connecting points) derived from Equation 2.

groups that were presented only one of the domains, either Squares 1-12 or Circles 13-24. These ratings are strikingly similar to the ratings by the experimental groups (Figure 1). Although the control groups employ a slightly greater range of categories (most notably, lower ratings of the smallest circles), it is clear that the presence of a second domain had only a small effect for the experimental conditions. Even the control groups rated the squares as though comparing them with sizes larger than any of those presented and the circles as though there were additional, smaller circles.

Figure 4 shows the ratings for two additional control conditions. The one labeled "No Gap 1-24" consisted of the double-rectangular combination of squares and circles, presented under instructions to ignore the differences in shape. Most subjects in this condition either gave the smallest circle a higher rating than the largest square or else rated it only a category step or so smaller. However, a few established separate scales for the two domains, with very similar ratings for the corresponding stimuli (e.g., largest squares and largest circles getting similar ratings). This partial failure to integrate the domains accounts for the break in the function at the transition between squares and circles. It thus appears that there is some tendency to isolate domains, even when instructed not to.

Figure 4 also shows the ratings by the control subjects presented only the eight smallest squares and eight largest circles, again with equal frequency and instructions to ignore the differences in shape (labeled "Gap 1-8, 17-24"). In spite of the very large gap, with no sizes from the middle third of the log-physical range, 14 of the 35 subjects rated Square 8 with a higher category than Circle 17 . The differences between the ratings of the same stimuli in the No-Gap 1-24 and in the Gap 1-8, 17-24 conditions demonstrate that the gap encourages the separation of squares and circles into two separate domains, contrary to instructions.

\section{Fitting the Range-Frequency Model}

As shown in Figures 1 and 3, the largest square is rated more than a full category step higher in the positively skewed set than in the negatively skewed set. A similar difference occurs for the smallest circles. The magnitude of this difference is much greater than would be predicted using the simple

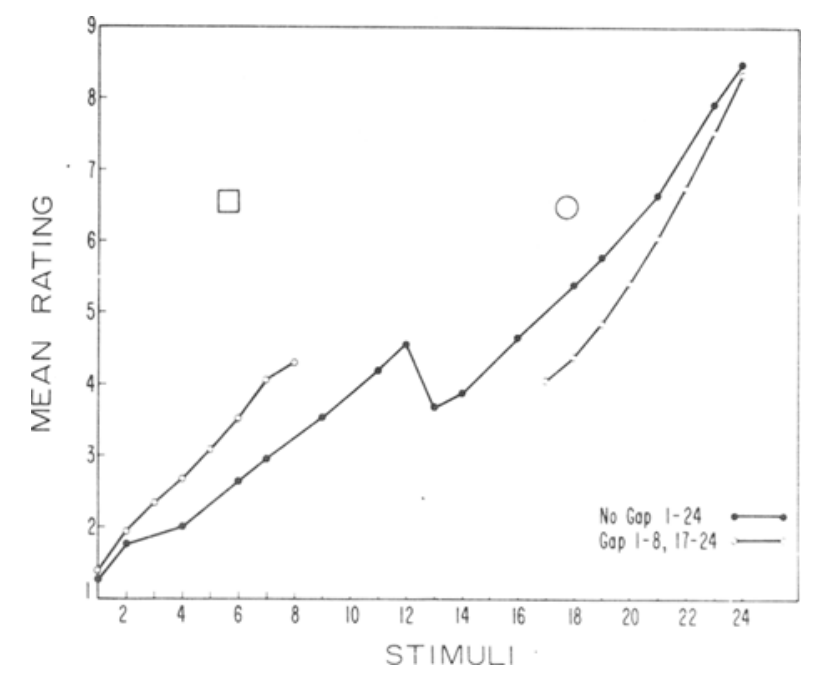

Figure 4. Integration of domains. Empirical ratings for two control conditions with instructions to ignore the differences in shape. Less integration when there is a large gap separating domains. 
Figure 5. Contextual effects within single domains. Empirical ratings (larger symbols) for three 6-17 sets of squares. Fitted functions (lines connecting points) derived from Equation 2 but in this case similar to fit from Equation 1.

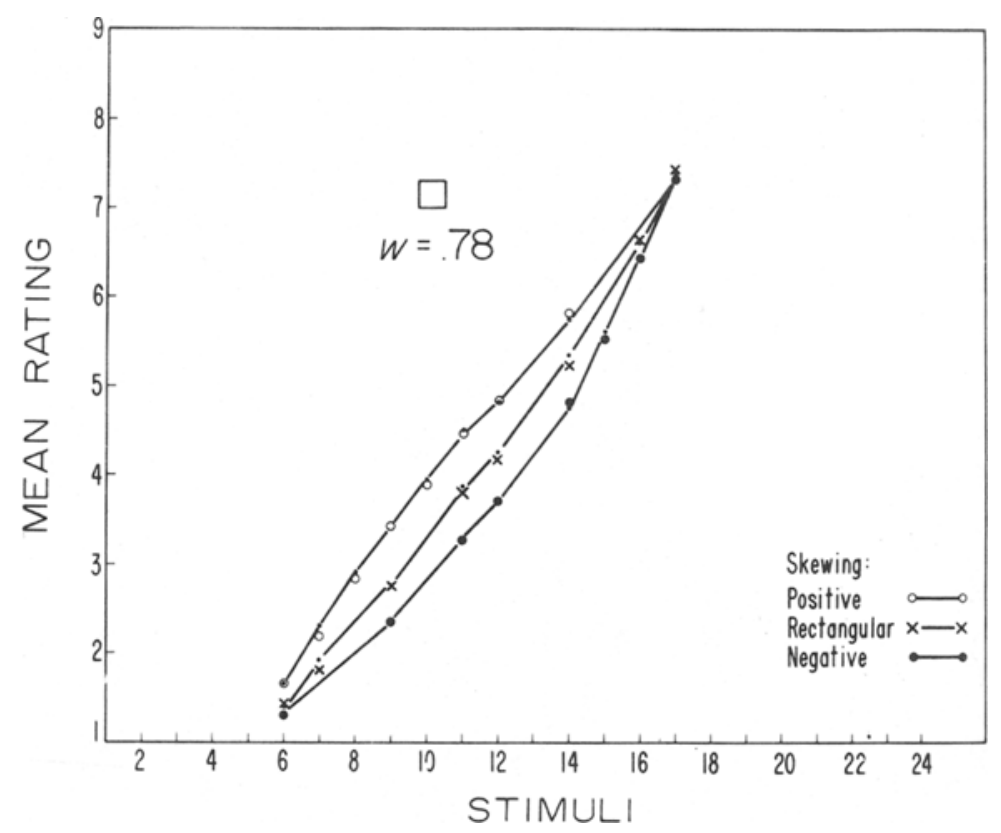

range-frequency model described for earlier data (Parducci, 1974; Parducci \& Perrett, 1971):

$$
J_{i d}=w R_{i}+(1-w) F_{j d},
$$

where $J_{i d}$ is the rating of the ith stimulus in the dth distribution, $F_{i d}$ is what this rating would have been if the same number of stimuli had been assigned to each category while maintaining an ordinal scale ( $F_{\text {id }}$ is calculated a priori by transforming the percentile rank of Stimulus $i$ linearly so that 0 and 100 become the extreme categories, 1 and 9 ), and $w$ is the relative weighting of the range value, $R_{i}$. The range value represents the position of the stimulus relative to the endpoints of the subjective range. In practice, range values have been inferred from empirical ratings of one distribution and used to predict the ratings of other distributions with the same physical endpoints. This assumes that $R_{i}$ is independent of the stimulus frequencies. But since $F_{\text {id }}$ differs by only half a category step for the largest square in the negative and positive distributions, the full-step difference in actual rating is inconsistent with this assumption.

One possibility is that the subjective endpoint is more extreme when stimuli are presented with greater frequency at that end of the series range. To describe this apparent effect, a post hoc fitting was employed in which the subjective range (as represented by the range values) was left free to vary for different distributions, with the restriction only that the range values of stimuli in any particular distribution are related linearly to the range values for the same stimuli in other distributions:

$$
\mathbf{J}_{\text {id }}=w\left(\beta_{\mathrm{d}} \mathbf{R}_{\mathbf{i}}+\alpha_{\mathrm{d}}\right)+(1-w) \mathrm{F}_{\mathrm{id}} .
$$

The lines in Figures 1, 3, and 5 were fitted using Equation 2 in an iterative program (Wickens, 1967) that minimized the squared differences between fitted and obtained ratings for the six stimuli common to all three distributions. Separate fits were obtained for each of the five sets of data from conditions in which the distribution was varied: ratings of squares in the experimental conditions, ratings of circles in the same conditions, ratings of squares and separately of circles in the first six control conditions, and ratings of Squares 6-17 which are shown in Figure 5.

The best-fit weightings of the range (the values of

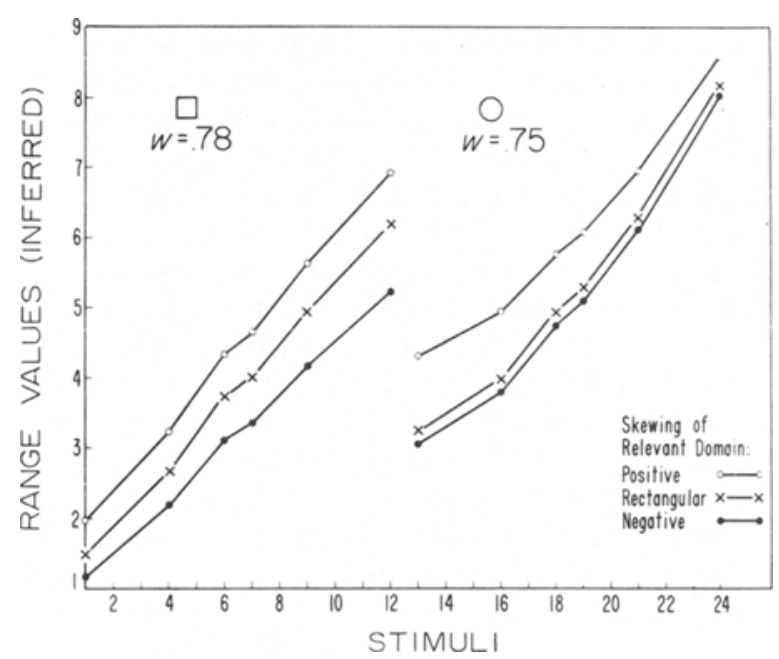

Figure 6. Range values derived from Equation 2 for experimental conditions. Values of $\alpha$ are $.66, .89$, and 1.35 for negatively skewed, rectangular, and positively skewed sets of squares, respectively; corresponding values for circles are $2.42,2.63$, and 3.77. Values of $\beta$ are $.51, .59$, and .62 for squares, .62, .61, and .53 for corresponding sets of circles. (These values are only unique up to a linear transformation.) 
w shown in Figures 1, 3, and 5) are all between .75 and .80 . This is well above the .5 obtained in the previous research with squares-although $w$ has been as high as .9 for practiced subjects (Parducci \& Perrett, 1967). It may be more difficult to keep track of the stimulus frequencies when the endpoints of the series are so far removed from the background anchors; as a consequence, the degree of skewing may have less effect on the ratings. The poorest predictions in the earlier research with squares were for those sets with the narrowest ranges (1-20 and 5-24, shown in Figure 9 of Parducci \& Perrett, 1971); when predictions are made using Equation 1 with $w=.8$ instead of .5 , the fit for these earlier narrowrange sets is greatly improved, the error variance being reduced by more than half.

The fits using Equation 2 yield transformed range values, $\beta_{\mathrm{d}} R_{\mathrm{id}}+\alpha_{\mathrm{d}}$, which can be used to describe how the subjective range depends on skewing. These transformed range values are plotted in Figure 6 for the six experimental conditions; similar plots were obtained for the corresponding control conditions (not shown). It is clear that the biggest effect of skewing is upon the range values of those stimuli furthest from the background anchors, such as Square 12 and Circle 13. For the three 6-17 conditions, there is essentially only one range function (not shown), i.e., the simple range-frequency model of Equation 1 provides an excellent fit.

\section{DISCUSSION}

It is surprising that ratings of each domain are so independent of the skewing of the other domain when the same set of physical responses is used in both domains. For, although circles are easily segregated from squares, it seems unlikely that subjects could so completely isolate their two sets of category frequencies, keeping separate counts of how often they had used the same categories in the two domains. Any confusion between these two sets of responses might have been expected to have systematic effects upon the ratings, raising them when the irrelevant set was skewed positively, lowering them when the skewing was negative. The failure to find any effect of the irrelevant domain suggests that the mechanism for judgment involves some kind of counting or representation of the more easily segregated stimulus frequencies rather than of response frequencies: subjects are putting the same number of stimuli in each category rather than using their alternative categories with equal frequency.

The modified range-frequency model of Equation 2 provides its close fit to the present data by allowing the subjective range to vary up to a linear transformation. The endpoints of the subjective range, as inferred from Equation 2 and by extrapolation from Figure 6 (to stimulus values corresponding to the two extreme categories), appear to depend upon four different factors: (1) the physical values of the two extreme stimuli presented for judgment, (2) background stimulus anchors, (3) the presence of irrelevant or unjudged stimuli, and (4) the skewness of the distribution of values presented for judgment.

The first of these factors, emphasized in range theories (e.g., Gravetter \& Lockhead, 1973; Upshaw, 1969; Volkmann, 1951), is well substantiated by research in which the physical values of the extreme stimuli were varied independently of other features of the stimulus distribution (Parducci, 1963; Parducci, Calfee, Marshall, \& Davidson, 1960; Parducci \& Perrett, 1971). It can also be inferred from the present data. For example, Square 6 is rated much lower in the 6-17 set (in which it is the smallest size) than in any of the 1-12 sets (in which it is near the middle of the range of sizes).

The second factor, background anchoring, is suggested by the lower ratings for squares than for circles when both occupy the same relative position in their respective sets. Even when circles are not presented, the largest square is rated only "Slightly Larger than Average" or "Large" on the 9-category scale, as though it were being compared with some larger size. The lighted background field against which the figures are projected may serve as a distant anchor, for it represents the largest stimulus that could be presented under the experimental arrangements. The effects of experimental manipulation of the area of this field in the earlier research with squares suggested that the upper subjective endpoint is a weighted average of the largest of the series stimuli and this background area (Parducci \& Perrett, 1971, pp. 443, 445). The ratings also seem to reflect a natural anchor for the lower endpoint, the smallest size that would be clearly detectable. This seems to be only slightly below Square 1 in the present research.

The third factor, the irrelevant domain, has a systematic but restricted effect on the range. The rating of the smallest circle is much lower when no squares are presented, but the largest square is rated only slightly higher without the circles. This asymmetry may reflect differences in the prominence of the background anchors: subjects seeing only the squares have the background field always before them as a distant upper endpoint; the distant lower endpoint (the smallest detectable size) might be less of an influence upon subjects seeing only the circles.

Previous applications of range-frequency theory have not been troubled by these first three factors because background and endpoint effects, being constant across conditions, were encompassed by the range values of Equation 1. However, the present evidence for a fourth factor, the effect of skewness upon the range values, argues for the linear transformation allowed by Equation 2. This transformation does not seem inconsistent with the basic range- 
frequency approach. The small but highly significant differences in curvature associated with skewness (the quadratic component of the functions in Figure 1) are predicted accurately from the a priori frequency values. These predictions employ only a single empirical parameter, $w$, whose best-fit value is remarkably constant across the five computer fits to Equation 2.

Although providing assurance that subjects tend to assign the same number of stimuli to each category within each domain, the present data encourage a revision in the way the subjective range has been conceived: presenting one end of the stimulus series with higher relative frequency suggests to the subject the possibility of presentations that are still more extreme, so that his subjective range extends further beyond the endpoint of the regular series, particularly when this endpoint is very much less extreme than the background anchor. The subjective range is specified for each condition by the values of $\alpha$ and $\beta$ obtained from the computer fits to Equation 2. For experimental and also for control conditions, $\alpha$ is always lowest with negative skewing, highest with positive skewing; the same ordering is found for $\beta$ with the squares but is reversed for the circles. What this means is illustrated by the inferred range functions plotted in Figure 6. It is clear from these (and from the similar range functions for the corresponding control conditions, not shown) that the lower subjective endpoint for squares, corresponding to a range value of 1 , is slightly smaller than the smallest square, regardless of skewness. Similarly, the upper subjective endpoint for circles is always only slightly larger than the largest circle. These were the extreme values used in previous research with squares in which the subjective endpoints were just a bit more extreme than the most extreme of the stimuli actually presented.

What is new is that these range functions vary with skewness, indicating greater upward extension of the subjective range for the negatively skewed squares and greater downward extension for the positively skewed circles. Although the particular values of $\alpha$ and $\beta$ would apply only to the present experimental conditions, the general effects are symmetrical and orderly across the two domains. Furthermore, they seem consistent with our intuitive notions of how the subjective range is established.

For example, consider a salesman whose daily commissions vary between $\$ 0$ and $\$ 100$. His subjective range of incomes would extend much higher if he earned close to $\$ 100$ on most days than if he earned that much only on rare occasions. However, his subjective range would extend further (i.e., it would take relatively more to satisfy him) if he had reason to believe that he could be earning $\$ 200$ than if there were factors limiting his possible earnings to $\$ 125$.

Although this experiment demonstrates that sub- jects can establish independent contexts for different sets of stimuli varying on the same dimension (size), presented on alternate trials of the same short experimental session, and rated using the same nine categories, this ability to separate domains does not imply that they will in fact be separated. The separation of circles from squares was governed primarily by instructions. Those control subjects instructed to ignore the differences in shape were able, for the most part, to combine squares and circles into a single context so that the smaller circles were rated as large or larger than the largest squares.

However, many subjects did establish separate scales when there was a large gap in size between the smallest circle and largest square-even though instructed not to. A similar phenomenon appears in anchoring studies in which a single extreme stimulus may have little or no effect on the scale when it is too different from the regular series of stimuli on the dimension of judgment (Rogers, 1941; Sarris, 1967) or when it also differs on other dimensions (Brown, 1953). The present research shows that this segregation of contexts can occur even when subjects are using the same set of categories to describe different sets of stimuli.

\section{REFERENCES}

Brown, D. R. Stimulus-similarity and the anchoring of subjective scales. A mericun Juurnal of Psychology. 1953, 66. 199-214.

Gravetter, F., \& LockheaD, G. R. Criterial range as a frame of reference for stimulus judgment. Psychological Review, 1973, 80, 203-216.

Parducci, A. Range-frequency compromise in judgment. Psychological Monographs, 1963, 77(2, Whole No. 565).

Parducci, A. Category judgment: A range-frequency model. Psychological Review, 1965, 72, 407-418.

PARDucci, A. Contextual effects: A range-frequency analysis. In E. C. Carterette \& M. P. Friedman (Eds.), Handbook of perception (Vol. II). New York: Academic Press, 1974.

Parducci, A., Calfee, R. C., Marshall, L. M.. \& Davidson, L. P. Context effects in judgment: Adaptation level as a function of the mean, midpoint, and median of the stimuli. Joumal of Experimental Psychology, 1960, 60, 65-77.

Parducci, A., \& Perret, L. R. Contextual effects for category judgments by practiced subjects. Psychonomic Science, 1967. 9. 357-358.

Parducci, A., \& Perret, L. R. Category rating scales: Effects of relative spacing and frequency of stimulus values. Journal of Experimental Psychology Monograph, 1971, 89, 427-452.

Rogers, S. The anchoring of absolute judgments. Archives of Psychology, 1941, 37 (No. 261), 1-42.

SARRIS, V. Adaptation-level theory: Two critical experiments on Helson's weighted-average model. American Joumal of Psychology, 1967, 80, 331-344.

Upshaw, H. S. The personal reference scale: An approach to social judgment. In L. Berkowitz (Ed.), Advances in experimental social psychology (Vol. 4). New York: Academic Press, 1969.

Volkmann, J. Scales of judgment and their implications for social psychology. In J. H. Rohrer \& M. Sherif (Eds.), Social psychology at the crossroads. New York: Harper, 1951.

Wickens, T. D. Parameter estimation in Markov chain learning models. Unpublished Master's Thesis, Brown University, 1967.

(Received for publication May 12, 1976; revision accepted August 20, 1976.) 